6-10September

2010,

GiardiniNaxos,

Italy

\section{IAU Symposium}

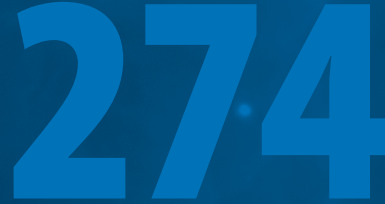

6-10 September 2010

Giardini Naxos, Italy

\title{
Advances in Plasma
} Astrophysics

\section{Advances}

in Plasma

Astrophysics

Edited by

\section{Bonanno \\ de Gouveia \\ Dal Pino}

Kosovichev

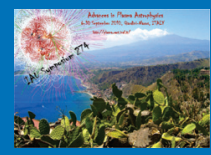

Proceedings of the International
Astronomical Union

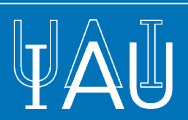

CAMBRIDGE
ISSN 1743-9213

International Astronomical Union

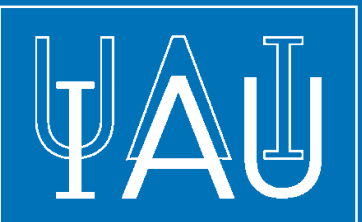

CAMBRIDGE UNIVERSITY PRESS
Alfio Bonanno

Elisabete de Gouveia Dal Pino

Alexander G.Kosovichev

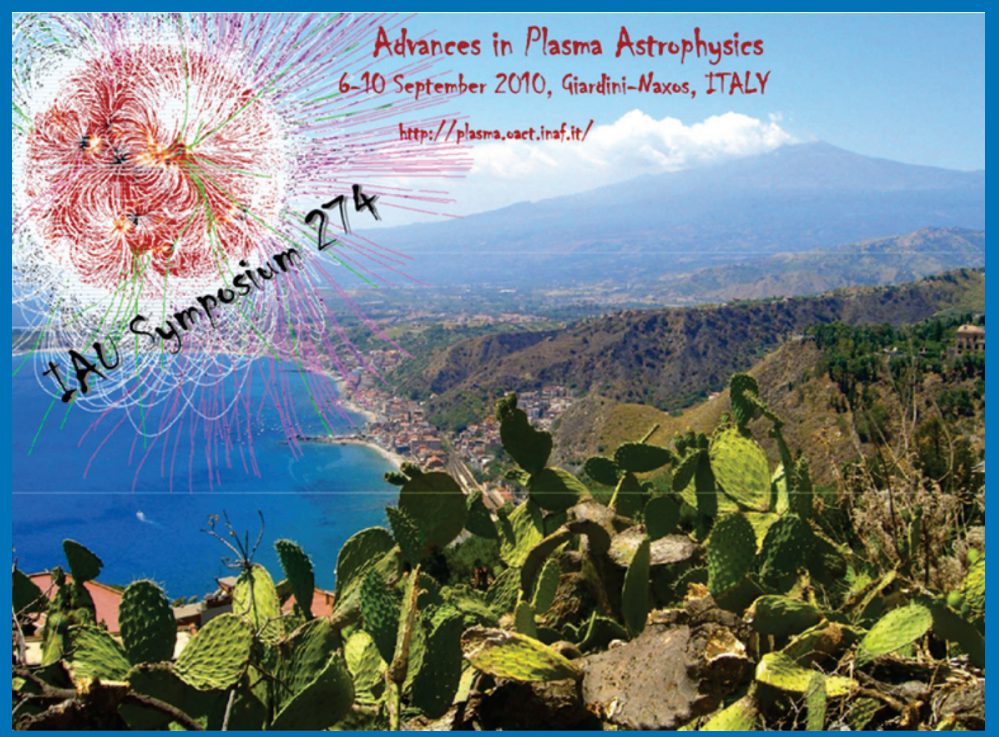


ADVANCES IN PLASMA ASTROPHYSICS

IAU SYMPOSIUM No. 274

COVER ILLUSTRATION: VIEW OF GIARDINI NAXOS, ITALY 


\title{
IAU SYMPOSIUM PROCEEDINGS SERIES 2010 EDITORIAL BOARD
}

\author{
Chairman \\ THIERRY MONTMERLE, IAU Assistant General Secretary \\ Laboratoire d'Astrophysique, Observatoire de Grenoble, \\ 414, Rue de la Piscine, Domaine Universitaire, \\ BP 53, F-38041 Grenoble Cedex 09, FRANCE \\ thierry.montmerle@obs.ujf-grenoble.fr
}

\author{
Advisers \\ IAN F. CORBETT, IAU General Secretary, \\ European Southern Observatory, Germany \\ UTA GROTHKOPF, European Southern Observatory, Germany \\ CHRSITIAAN STERKEN, University of Brussels, Pleinlaan 2, 1050 Brussels, Belgium
}

\section{Proceedings Editors}

IAUS 269: Galileo's Medicean Moons: their impact on 400 years of Discovery

Cesare Barbieri, Università di Padova, Dipto di Astronomia, Vicolo dell'Osservatorio 2, IT 35122 Padova, Italy

IAUS 270: Computational star formation

João Alves, Calar Alto Observatory, Centro Astronómico Hispano Alemán, c/ Jesus Durban Remon 2-2, ES 04004 Almeria, Spain

IAUS 271: Astrophysical dynamics: from stars to galaxies

Allan Sacha Brun, CEA/DSM/IRFU, Service d'Astrophysique, CEA Saclay, FR 91191 Gif-sur-Yvette, France

IAUS 272: Active OB stars: structure, evolution, mass loss, and critical limits

Coralie Neiner, GEPI, Observatoire Paris-Meudon, 5 place Jules Janssen, FR 92195 Meudon Cedex, France

IAUS 273: Physics of Sun and star spots

Debi Prasad Choudhary, CSUN, Physics-Astronomy Dept., 18111 Nordhoff St, Northridge, CA 91330-8268, USA

IAUS 274: Advances in plasma astrophysics

Alfio Bonanno, INAF, Osservatorio Astrofisico di Catania, Via S. Sofia 78, IT 95123 Catania, Italy

IAUS 275: Jets at all scales

Gustavo E. Romero, Instituto Argentino de Radioastronomía, CC 5, AR Villa Elisa (Bs As) 1894, Argentina

IAUS 276: The Astrophysics of planetary systems: formation, structure, and dynamical evolution

Alessandro Sozzetti, INAF, Osservatorio Astronomico di Torino, Strada Osservatorio 20, IT 10025 Pino Torinese, Italy

IAUS 277: Tracing the ancestry of galaxies (on the land of our ancestors)

Claude Carignan, Université de Montréal, Dept. de Physique, CP 6128 Succ. A, CA Montréal QC H3C 3J\%, Canada 
INTERNATIONAL ASTRONOMICAL UNION

UNION ASTRONOMIQUE INTERNATIONALE

International Astronomical Union

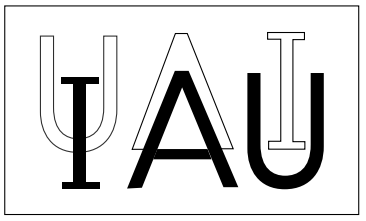

ADVANCES IN

\section{PLASMA ASTROPHYSICS}

\section{PROCEEDINGS OF THE 274th SYMPOSIUM OF THE INTERNATIONAL ASTRONOMICAL UNION HELD IN GIARDINI NAXOS, ITALY SEPTEMBER 6-10, 2010}

Edited by

ALFIO BONANNO

INAF-Osservatorio Astrofisico di Catania, Italy

ELISABETE DE GOUVEIA DAL PINO

Instituto Astronômico Geofisico - Universidade de São Paulo, Brasil

and

ALEXANDER G. KOSOVICHEV

Stanford University, USA 
CAMBRIDGE UNIVERSITY PRESS

The Edinburgh Building, Cambridge CB2 2RU, UnitedKingdom

40 West 20th Street, New York, NY 10011-4211, USA

10 Stamford Road, Oakleigh, Melbourne 3166, Australia

(C) International Astronomical Union 2011

This book is in copyright. Subject to statutory exception and to the provisions of relevant collective licensing agreements, no reproduction of any part may take place without the written permission of the International Astronomical Union.

First published 2011

Printed in the United Kingdom at the University Press, Cambridge

Typeset in System $\mathrm{HT}_{\mathrm{EX}} 2_{\varepsilon}$

A catalogue record for this book is available from the British Library

Library of Congress Cataloguing in Publication data

This journal issue has been printed on FSC-certified paper and cover board. FSC is an independent, non-governmental, not-for-profit organization established to promote the responsible management of the world.s forests. Please see www.fsc.org for information.

ISBN 9780521197410 hardback

ISSN 1743-9213 


\section{Table of Contents}

Preface .................................... xiii

Alfio Bonanno, Alexander Kosovichev and Elisabete de Gouveia Dal Pino, editors and co-chairs $S O C$

Organizing committee $\ldots \ldots \ldots \ldots \ldots \ldots \ldots \ldots \ldots \ldots \ldots \ldots \ldots \ldots \ldots \ldots \ldots$

Conference photograph $\ldots \ldots \ldots \ldots \ldots \ldots \ldots \ldots \ldots \ldots \ldots \ldots \ldots \ldots \ldots \ldots \ldots \ldots \ldots$

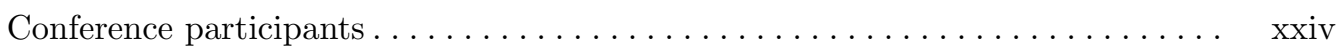

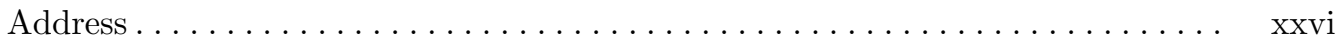

Gianni Strazzulla, Director of Catania Astrophysical Observatory

\section{Plasma astrophysics in laboratory}

The magnetized universe: its origins and dissipation through acceleration and leak-

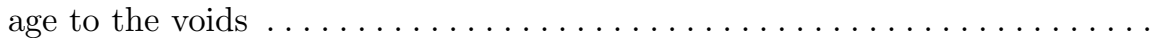

S. A. Colgate, H. Li, P. P. Kronberg

Study of magnetic reconnection in collisional and collisionless plasmas in Magnetic

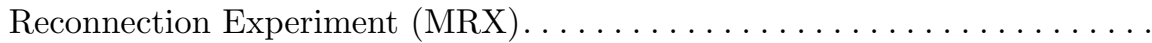
M. Yamada, H. Ji

Current status and future prospects for laboratory study of angular momentum transport relevant to astrophysical disks $\ldots \ldots \ldots \ldots \ldots \ldots \ldots$ H. Ji

Laboratory simulations of astrophysical jets $\ldots \ldots \ldots \ldots \ldots \ldots \ldots$

S. V. Lebedev, F. Suzuki-Vidal, A. Ciardi, M. Bocchi, S. N. Bland, G. Burdiak, J. P. Chittenden, P. de Grouchy, G. N. Hall, A. Harvey-Thompson, A. Marocchino, G. Swalding, A. Frank, E. G. Blackman, M. Camenzind

Laboratory-generated coronal mass ejections. . . . . . . . . . . . . .

C. Watts, Y. Zhang, A. Lynn, W. Manchester, N. Arge

Region-1 field aligned currents in experiments on laser-produced plasma interacting with magnetic dipole . ......................

I. F. Shaikhislamov, Yu P. Zakharov, V. G.Posukh, E. L. Boyarintsev,

A. V. Melekhov, V. M. Antonov, A. G. Ponomarenko

Calculation of fusion rates at extremely low energies in laser plasmas

D. Mascali, N. Gambino, S. Tudisco, A. Anzalone, A. Bonanno,

S. Gammino, F. Musumeci

\section{Interstellar, space and planetary plasmas}

A new viscous instability in weakly ionised protoplanetary discs $\ldots \ldots \ldots \ldots$. A. Johansen, M. Kato, T. Sano

Magnetic fractures or reconnection of type II

G. Haerendel 
Particle Acceleration in Fast Magnetic Reconnection . . . . . . . . . . . . . .

A. Lazarian, G. Kowal, E. de Gouveia Dal Pino, E. Vishniac

Weakly Imbalanced Strong Turbulence . . . . . . . . . . . . . . . .

A. Beresnyak

Plasma astrophysics implication in discovery and interpretation of X-ray radiation

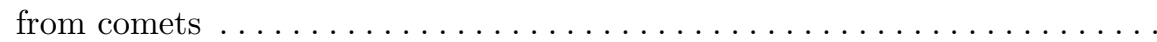

S. Ibadov

Large eddy simulations in plasma astrophysics. Weakly compressible turbulence in local interstellar medium ........................

A. A. Chernyshov, K. V. Karelsky, A. S. Petrosyan

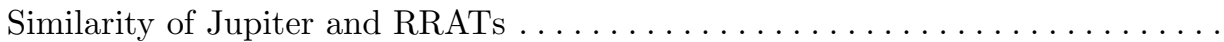

I. Gezer, R. Pekünlü

On the development of a Spherical Hybrid Model -Lessons and applications....

S. Dyadechkin, E. Kallio, R. Jarvinen, P. Janhunen, V.S. Semenov, H.K.

Biernat

Solar plasma generated by sungrazing comets $\ldots \ldots \ldots \ldots \ldots \ldots \ldots$

F. S. Ibodov, S. Ibadov

Development of the PFO-CFO hypothesis of Solar System formation: Why do the celestial objects have different isotopic ratios for some chemical elements? .

E. A. Kadyshevich, V. E. Ostrovskii

A note on using thermally driven solar wind models in MHD space weather simu-

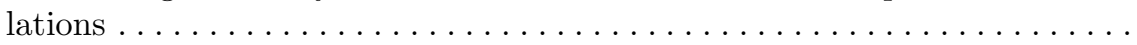

J. Pomoell, R. Vainio

\section{Solar and stellar plasma}

Anomalous momentum transport in astrophysical return-current beam plasmas -the two-dimensional electromagnetic case $\ldots \ldots \ldots \ldots \ldots \ldots$ K.W. Lee, J. Büchner

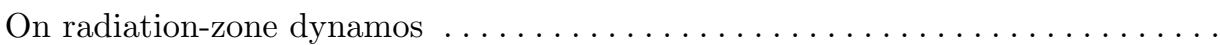

G. Rüdiger, M. Gellert, R. Arlt

Emergence of intermittent structures and reconnection in MHD turbulence ....

A. Greco, S. Servidio, W.H. Matthaeus, P. Dmitruk

Realistic MHD simulations of magnetic self-organization in solar plasma. . . . . .

I. N. Kitiashvili, A. G. Kosovichev, A. A. Wray, N. N. Mansour

Helicity transport in a simulated coronal mass ejection $\ldots \ldots \ldots \ldots \ldots \ldots$

B. Kliem, S. Rust, N. Seehafer

Coupled Alfvén and kink oscillations in an inhomogeneous corona . . . . . . . .

D. J. Pascoe, A. N. Wright, I. De Moortel

Weak turbulence theory of dispersive waves in the solar corona . . . . . . . .

F. Spanier, R. Vainio 
The Effect of Plume/Interplume Lanes on Ion-Cyclotron Resonance Heating ...

S. Doğan, E. R. Pekünlü

Small-scale flux emergence events observed by Sunrise/IMaX . . . . . . . . . . .

S. L. Guglielmino, V. Martínez Pillet, J. C. del Toro Iniesta, L. R. Bellot

Rubio, F. Zuccarello, S.K. Solanki, \& the Sunrise/IMaX team

Cross helicity in stellar magnetoconvection $\ldots \ldots \ldots \ldots \ldots \ldots \ldots$

M. Küker, G. Rüdiger

Some properties of prominence eruption associated CMEs during 1996-2009 . .

N. Mittal

Separation of solar radio bursts in a complex spectrum $\ldots \ldots \ldots \ldots \ldots \ldots$

H. Mészárosová, J. Rybák, M. Karlický, K. Jiřička

An hybrid neuro-wavelet approach for long-term prediction of solar wind . . . . .

C. Napoli, F. Bonanno, G. Capizzi

Exploiting solar wind time series correlation with magnetospheric response by using

an hybrid neuro-wavelet approach $\ldots \ldots \ldots \ldots \ldots \ldots \ldots \ldots \ldots \ldots \ldots \ldots \ldots$

C. Napoli, F. Bonanno, G. Capizzi

A shell model for turbulent dynamos . . . . . . . . . . . . . . .

G. Nigro, D. Perrone, P. Veltri

Energetic particle acceleration and transport by Alfvén/acoustic waves in tokamak-

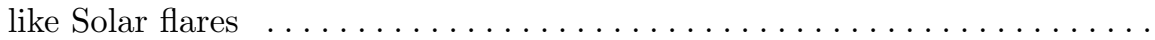

M. Obergaulinger, M. García-Muñoz

CME evolution and 3D reconstruction with STEREO Data ...........

A. Orlando, F. Zuccarello, P. Romano, FP. Zuccarello, M. Mierla, D.

Spadaro, R. Ventura

Hybrid Vlasov simulations for alpha particles heating in the solar wind.......

D. Perrone, F. Valentini, P. Veltri

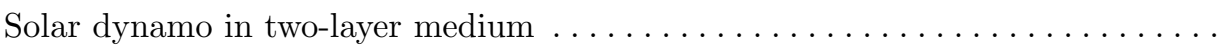

H. Popova

Modeling circumstellar envelope with advanced numerical codes . . . . . . . .

P. Procopio, A. De Rosa, C. Burigana, G. Umana, C. Trigilio

Energetic Solar Electrons - Whistler Bootstrap, Magnetic Knots and Small-scale

Reconnection ...................................

I. Roth

178

Solar flares: observations vs simulations. . . . . . . . . . . . . .

F. Rubio da Costa, F. Zuccarello, N. Labrosse, L. Fletcher, T. Prosecký,

J. Kašparová

A way to detect the magnetic helicity using the observable polarized radio emission R. Stepanov, A. Volegova

Magnetic helicity evolution inside a hexagonal convective cell $\ldots \ldots \ldots \ldots \ldots$

A. Smyrli, D. Mackay, F. Zuccarello 
Unveiling the Butterfly Diagram structure $\ldots \ldots \ldots \ldots \ldots \ldots \ldots \ldots$

M. Ternullo

Superdiffusive and ballistic propagation of protons in solar energetic particle events 198 E. M. Trotta, G. Zimbardo

Electron acceleration by a wavy shock front: details on angular distribution ... 201 M. Vandas, M. Karlický

Spatio-temporal variability of the photospheric magnetic field . . . . . . . . . 204

A. Vecchio, M. Laurenza, D. Meduri, V. Carbone, M. Storini

\section{Plasma around compact objects}

Plasma processes in pulsar magnetospheres. 208

D. B. Melrose

Collisionless shocks and particle acceleration: lessons from studies of heliospheric shocks

T. Terasawa

Special relativistic magnetohydrodynamic simulation of two-component outflow powered by magnetic explosion on compact stars . . . . . . . . . . J. Matsumoto, Y. Masada, E. Asano, K. Shibata

Alfvén resonance absorption in electron-positron plasmas

N. F. Cramer

Theory of quasi-stationary kinetic dynamos in magnetized accretion discs . . . . C. Cremaschini, J.C. Miller, M. Tessarotto

Stationary and axisymmetric configurations of compact stars with extremely strong and highly localized magnetic fields $\ldots \ldots \ldots \ldots \ldots \ldots \ldots \ldots \ldots \ldots \ldots \ldots$

K. Fujisawa, S. Yoshida, Y. Eriguchi

Kinetic closure conditions for quasi-stationary collisionless axisymmetric magneto-

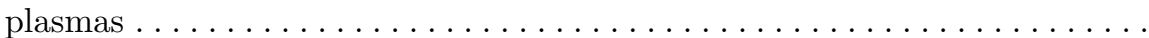

C. Cremaschini, J.C. Miller, M. Tessarotto

Ponderomotive barrier for plasma particles on the boundary of astrophysical jets A. A. Dubinova, V.V. Kocharovsky

GRB spectral parameter modeling

G. D. Fleishman, F.A. Urtiev

Magnetic collimation of relativistic jets: the role of the black hole spin . . . . . .

N. Globus, C. Sauty, V. Cayatte

Current instabilities in the pulsar magnetosphere $\ldots \ldots \ldots \ldots \ldots \ldots \ldots$

A. Jessner, H. Lesch, M. Krame

Electromagnetic emission by subsequent processes $\mathrm{L} \rightarrow \mathrm{L}^{\prime}+\mathrm{S}$ and $\mathrm{L}+\mathrm{L}^{\prime} \rightarrow \mathrm{T} \ldots$ M. Karlický M. Bárta

Quasi-periodic oscillations in solar X-ray sources $\ldots \ldots \ldots \ldots \ldots \ldots$

H. Mészárosová, M. Karlický, F. Fárník 
Two component relativistic acceleration and polarized radiation of the parsec-scale

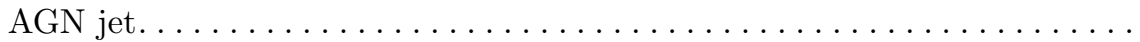

O. Porth

Particle acceleration in Blazars. . . . . . . . . . . . . . . . . . . .

M. Weidinger, F. Spanier

\section{Observational and modelling programs for plasma astrophysics}

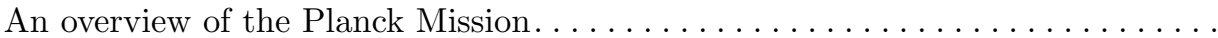

N. Mandolesi, C. Burigana, A. Gruppuso, P. Procopio, S. Ricciardi on behalf of the Planck Collaboration

Towards observational MHD. Advances in spectropolarimetry and the prospects

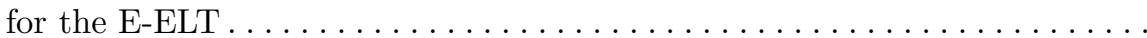

K. G. Strassmeier

New interactive solar flare modeling and advanced radio diagnostics tools. . . . . . 280

G. D. Fleishman, G.M. Nita, D.E. Gary

ALMA and solar research 284 M. Karlický, M. Bárta

Investigations of solar plasma in the interior and corona from solar dynamics observatory

A. G. Kosovichev

Inclusion of velocity gradients in the Unno solution for magnetic field diagnostic from spectropolarimetric data . . . . . . . . . . . . . . . . .

G. Molodij, V. Bommier

Solar wind turbulence: Advances in observations and theory............ 295 J.J. Podesta

"Ambipolar diffusion" and magnetic reconnection 302 Y. T. Tsap, A. V. Stepanov

Plasmoid ejections driven by dynamo action underneath a spherical surface.... J. Warnecke, A. Brandenburg, D. Mitra

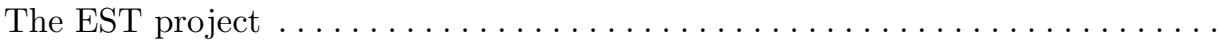

F. Zuccarello and the EST team

Optimized gyrosynchrotron algorithms and fast codes . .

A. A. Kuznetsov, G. D. Fleishman

\section{Plasmas in galaxies and galaxy cluster}

Development of the theory of instabilities of differentially rotating plasma with astrophysical applications

J. G. Lominadze 
Magnetism in galaxies - Observational overview and next generation radio tele-

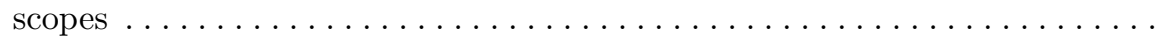

R. Beck

MHD turbulence-Star Formation Connection: from pc to kpc scales . . . . . . . .

E.M. de Gouveia Dal Pino, R. Santos-Lima, A. Lazarian, M.R.M. Leão, D. Falceta-Gonçalves, G. Kowal

Relativistic plasma and ICM/radio source interaction $\ldots \ldots \ldots \ldots \ldots \ldots \ldots$

L. Feretti, G. Giovannini, F. Govoni, M. Murgia

Supernova-driven interstellar turbulence and the galactic dynamo . . . . . . . .

O. Gressel, D. Elstner, G. Rüdiger

Cosmic-ray driven dynamo in galaxies

M. Hanasz, D. Wóltanski, K. Kowalik and H. Kotarba

On the solution of the Kompaneets equation in cosmological context: a numerical code to predict the CMB spectrum under general conditions. . . . . . . . . .

C. Burigana, P. Procopio, A. De Rosa

Statistical tools of interstellar turbulence: connecting observations with theory .

B. Burkhart, A. Lazarian

An XMM-Newton view of a small sample of Seyfert 1 Galaxies . . . . . . . . .

M. V. Cardaci, G. F. Hagele, M. Santos-Lleó, Y. Krongold, A. I. Díaz, P. Rodriguez-Pascual

How can vorticity be produced in irrotationally forced flows? . . . . . . . . . .

F. Del Sordo, A. Brandenburg

The fate of magnetic fields in colliding galaxies $\ldots \ldots \ldots \ldots \ldots \ldots$

H. Kotarba, H. Lesch, K. Dola, T. Naab

3D numerical simulations of magnetic field evolution in barred galaxies and in spiral galaxies under influence of tidal forces $\ldots \ldots \ldots \ldots \ldots \ldots \ldots$

K. Otmianowska-Mazur, K. Kulpa-Dybel, B. Kulesza-Żydzik, H. Siejkowski, G. Kowal

Ferromagnetic properties of charged vector bosons condensate in the early universe G. Piccinelli

3D model of magnetic fields evolution in dwarf irregular galaxies . . . . . . . .

H. Siejkowski, M. Soida, K. Otmianowska-Mazur, M. Hanasz, D. J. Bomans

General relativistic magnetic perturbations and dynamo effects in extragalactic

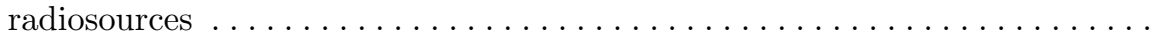

L. C. G. de Andrade

Cosmic ray driven dynamo in barred and ringed galaxies $\ldots \ldots \ldots \ldots \ldots \ldots$

K. Kulpa-Dybel, K. Otmianowska-Mazur, B. Kulesza-Żydzik, G.Kowal, D. Wóltański, M. Hanasz, K. Kowalik 


\section{Plasma astrophysics in numerical simulations}

Simulations of astrophysical dynamos $\ldots \ldots \ldots \ldots \ldots \ldots \ldots \ldots \ldots$

A. Brandenburg

Relativistic jets and current driven instabilities

A. Ferrari, A. Mignone, M. Campigotto

Global MHD simulations of disk-magnetosphere interactions: accretion and out-

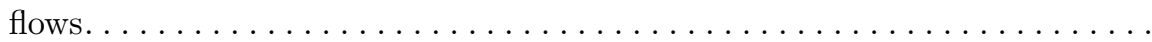

M. M. Romanova, R. V. E. Lovelace, G. V. Ustyugova, A. V. Koldoba

Recent results from simulations of the magnetorotational instability . . . . . . . J. M. Stone

Numerical study of jets produced by conical wire arrays on the Magpie pulsed

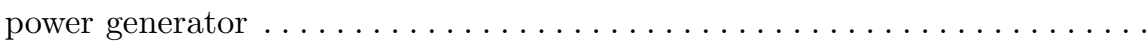
M. Bocchi, J. P. Chittenden, A. Ciardi, F. Suzuki-Vidal, G. N. Hall, P. de Grouchy, S. V. Lebedev, S. C. Bott

Three dimensional simulations of Hall magnetohydrodynamics . . . . . . . . . . D. O. Gómez

Stationary and axisymmetric magnetized equilibria of stars and winds ....... S. Yoshida, K. Fujisawa, Y. Eriguchi, S. Yoshida, R. Takahashi

Shock refraction from classical gas to relativistic plasma environments . . . . . . R. Keppens, P. Delmont, Z. Meliani

Magnetic field amplification by relativistic shocks in a turbulent medium ...... Y. Mizuno, M. Pohl, J. Niemiec, B. Zhang, K.-I. Nishikawa, P. E. Hardee

Saturation of MRI via parasitic modes M. E. Pessah

Formation of electron clouds during particle acceleration in a $3 \mathrm{D}$ current sheet . V. V. Zharkova, T. Siversky

3D turbulent reconnection driven current-sheet dynamics: solar applications ... L. Bettarini, G. Lapenta

Decay of trefoil and other magnetic knots .................. S. Candelaresi, F. Del Sordo, A. Brandenburg

Magnetic helicity fluxes in $\alpha \Omega$ dynamos. . . . . . . . . . . . .

S. Candelaresi, A. Brandenburg

A first model of stable magnetic configuration in stellar radiation zones . . . . . . V. Duez, J. Braithwaite, S. Mathis

Kinetic Simulations of Type II Radio Burst Emission Processes . . . . . . . . . . U. Ganse, F. Spanier, R. Vainio

Turbulent magnetic pressure instability in stratified turbulence . . . . . . . . .

K. Kemel, A. Brandenburg, N. Kleeorin, I. Rogachevskii

Current-Driven Kink Instability in Relativistic Jets . . . . . . . . . . . . . .

Y. Mizuno, P. E. Hardee, Y. Lyubarsky, K.-I. Nishikawa 
High-order methods for the simulation of hydromagnetic instabilities in core-collapse

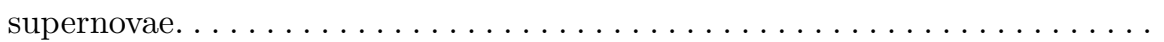

T. Rembiasz, M. Obergaulinger, M. Angel Aloy, P. Cerdá-Durán, E. Müller

Dynamo in the Intra-Cluster Medium: Simulation of CGL-MHD Turbulent Dy-

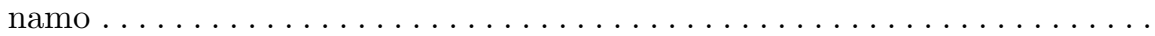
R. Santos-Lima, E. M. de Gouveia Dal Pino, A. Lazarian, G. Kowal, D. Falceta-Gonçalves

Author index 485

Object index . 488 


\section{Preface}

The organization of this Symposium was first motivated by the fact that nowadays connecting astrophysical theory, observations, simulations and laboratory astrophysics is widely appreciated by the scientific community. In this respect this symposium was an important occasion to discuss recent observational, theoretical and experimental efforts in understanding the basic plasma processes in the Universe, with broad synergies among many areas of astrophysics, including the origin and dynamics of magnetic fields in astrophysical systems (the dynamo problem), the origin of x-ray emitting coronas and the role of magnetic reconnection, acceleration of charged particles, winds and jets from highly-evolved stars and supernova remnants, plasma radiation processes, turbulence of the magnetized plasma in astrophysical objects and in the interstellar and intergalactic media and the solar wind, quantum plasmas under extreme conditions in planetary interiors and in exotic stars, and other key problems in modern plasma astrophysics.

The most important goal of the symposium was therefore to bring together experts from plasma physics, MHD, laboratory experiments and numerical simulation communities. In fact, plasma astrophysicists have always been a fairly small group, often distinct from the main astrophysical community, holding their own workshops and special sessions at plasma physics conferences. Despite the identification of a rich class of physical problems of mutual interest, the plasma physics and astrophysics communities remain, for the most part, quite detached, with different societies and memberships, conferences and journals. This Symposium contributed to promote links and cooperation between these communities, to discuss the recent advances in understanding the fundamental plasma physics processes and their application to interpretation and understanding of phenomena observed in astrophysical plasmas at various scales. Despite the wide range of temporal and spatial scales and conditions the basic physics of these phenomena is often very similar. Therefore, it was a unique occasion to discuss these issues together.

Undoubtedly, such discussions and exchange of ideas from different fields have led to a better understanding of the basic mechanisms of many observational phenomena, their origin, structure and dynamics, and will guide future astrophysical observing programs, as well as theoretical and numerical modeling and laboratory experiments in plasma astrophysics. Such interdisciplinary and cross-discipline discussions become increasingly important as they provide a special opportunity to get a broader view of the field and new ideas about methodologies and approaches. This aspect is particularly crucial for younger researchers because the learning curves in various sub-disciplines become steeper and steeper. For this reason during the Symposyum, in addition to traditional review and contributed talks covering outstanding observational and theoretical problems of astrophysical plasmas, considerable time was devoted to exciting discussions at the end of each day session.

We would like to dedicate this meeting to Stirling Colgate, Gerhard Haerendel, Jumber Lominadze, Don Melrose, and Lucio Paternò, who made outstanding contributions to the field of plasma astrophysics.

It is also a great pleasure to acknowledge the financial support of our sponsors listed on page $x v i$ of these Proceedings and the active support of the members of the LOC for performing so efficiently and enthusiastically the numerous tasks always associated with such a big meeting. In particular, our sincere thanks go to his competent and patient approach of Christian Napoli who helped the participants solving technical/computer problems, Gabriella Caniglia, Fatima Rubio da Costa and Enrico Corsaro who took care of the 
xiv

logistics of participants, Paolo Romano for his editorial work, to Elisabetta Palumbo, Luigia Santagati, Corrado Trigilio and Grazia Umana for assisting the participants in their numeous needs.

We also aknowledge the professional contribution made by Rainer Arlt who took the photos we published in this volume.

Finally, a special mention must be given to Daniela Recupero, whose professional skills and human gifts have been essential ingredients for the success of this meeting.

Unfortunately, a sad news arrived while we were finishing the editing of this volume, which we cannot help referring. Ilkka Tuominen, a close friend, a brilliant scientist and a mentor for many who attended the meeting, passed away in March 2011, leaving us astonished but at the same time grateful for the great heritage of human and scientific talents he left us. He attended this meeting with his usual enthusiasm and curiosity, providing the LOC with moral support and nice jokes.

We will all miss him. Ciao Ilkka.

Alfio Bonanno, Alexander Kosovichev and Elisabete de Gouveia Dal Pino, editors and co-chairs $S O C$ 


\section{THE ORGANIZING COMMITTEE}

\section{Scientific}

G.Belvedere (Italy)

A. Bonanno (co-chair Italy)

A. Brandenburg (Sweden)

E. de Gouveia Dal Pino (co-chair Brasil)

M. Goossens (Belgium)

G.Haerendel (Germany)

H. Ji (USA)

A. Kosovichev (co-chair USA)
K. Otmianowska-Mazur (Poland)

R. Rosner (USA)

M. Shats (Australia)

K. Shibata (Japan)

L. Vlahos (Greece)

D. Wu (China)

L. Zeleny (Russia)

\section{Local}

G. Belvedere

P. Romano

A. Bonanno (chair)

L. Santagati

G. Caniglia

C. Trigilio

S. Gammino

S. Tudisco

M.E. Palumbo

G. Umana

D. Recupero

\section{Acknowledgements}

The symposium is sponsored and supported by the IAU Divisions IV (Stars), VI (Interstellar Matter), VII (Galactic System), VIII (Galaxies) and XI (Space and High Energy Astrophysics); and by the IAU Commissions No. 26 (Binary and Multiple Stars), No. 28 (Galaxies), No. 29 (Stellar Spectra), No. 34 (Interstellar Matter), No. 35 (Stellar Constitution), No. 36 (Theory of Stellar Atmospheres), No. 37 (Star Clusters and Associations) and No. 44 (Space and High Energy Astrophysics).

Funding by the

International Astronomical Union, Istituto Nazionale di Astrofisica,

Dipartimento di Fisica ed Astronomia dell'Universitá degli Studi di Catania, Istituto nazionale di Fisica Nucleare-Sezione di Catania,

Laboratorio Nazionale del Sud-Sezione di Catania,

European Physical Society,

European Science Foundation 


\section{Stirling Colgate}

In 2010 Stirling Colgate has turned 85. His career has spanned more than 60 years, starting as a PhD student in physics at Cornell University and working at Lawrence Livermore and New Mexico Institute of Mining and Technology. After the success of Bravo Test in 1950s, the first deliverable thermonuclear bomb, he was encouraged to begin research on thermonuclear fusion and plasma physics. Many of his scientific successes, however, have been realised at the Los Alamos National Laboratory where he arrived in 1976, joining the Theoretical Division.

$\mathrm{He}$ is recognized for negotiating the cessation of high-altitude and outer space nuclear tests. Colgate also has inspired the inertial fusion and astrophysics programs at Los Alamos and Lawrence Livermore and contributed basic science to fusion ignition and burn, plasma confinement and shock wave physics. In 2006 he has been awarded the Los Alamos Medal.

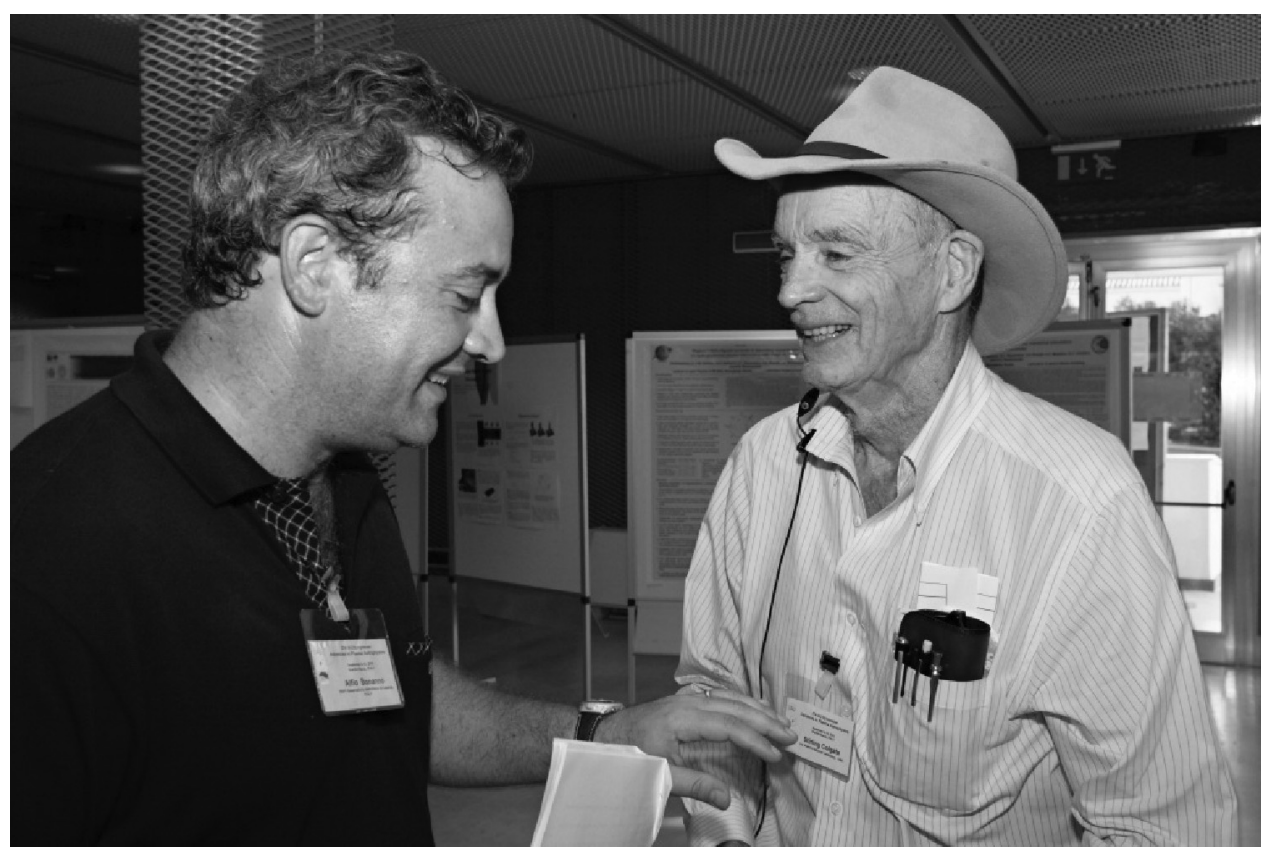

Stirling Colgate (right) and Alfio Bonanno 


\section{Jumber Georgievich Lominadze}

September 20, 2010, was the 80th birthday of Jumber Georgivich Lominadze, one of the leading plasma astrophysicists, founder of the Plasma Astrophysics Center in Georgia, Head of the Center for Space Research, and Academician of the Georgian National Academy of Sciences. Jumber Lominadze was born in 1930 in Tbilisi. After the graduation from Moscow University in 1955 he worked at the Russian (Ural) Nuclear Center. In 1958 he returned to Tbilisi, and actively participated in the development of plasma physics and nuclear fusion research at the Georgian Institute of Physics. His studies were focused on the propagation and absorption of cyclotron waves in plasma, and were published in book 'Cyclotron Waves in Plasma' (Metsnierba, Tbilisi, 1975; Pergamon Press, Oxford, 1981). In 1976 he founded the Plasma Astrophysics Center, which under his leadership became one of the leading world-class research center. He actively developed international collaborations, and organized a series of legendary conferences, workshops, and schools on plasma astrophysics, which play very important role in the development of this field. He developed the electromagnetic theory of electron-positron plasma, which was used to explain mechanisms and properties of Crab pulsar radiation in different bands and other fundamental processes. More recently, he and his colleagues studied the physics of accretion disks, jets, resonance transformation of oscillations, excitation of waves by vortices, dynamical processes in shearing flows and instabilities in rotating plasma. For more than 40 years he has been teaching at Tbilisi University, and supervised the research of more than $20 \mathrm{PhD}$ students.

His former students now form a core of the Georgian Plasma Astrophysics school. On behalf of the IAUS 274 participants we sincerely congratulate Professor Jumber Lominadze on his 80th birthday and wish him all the best for the coming years.

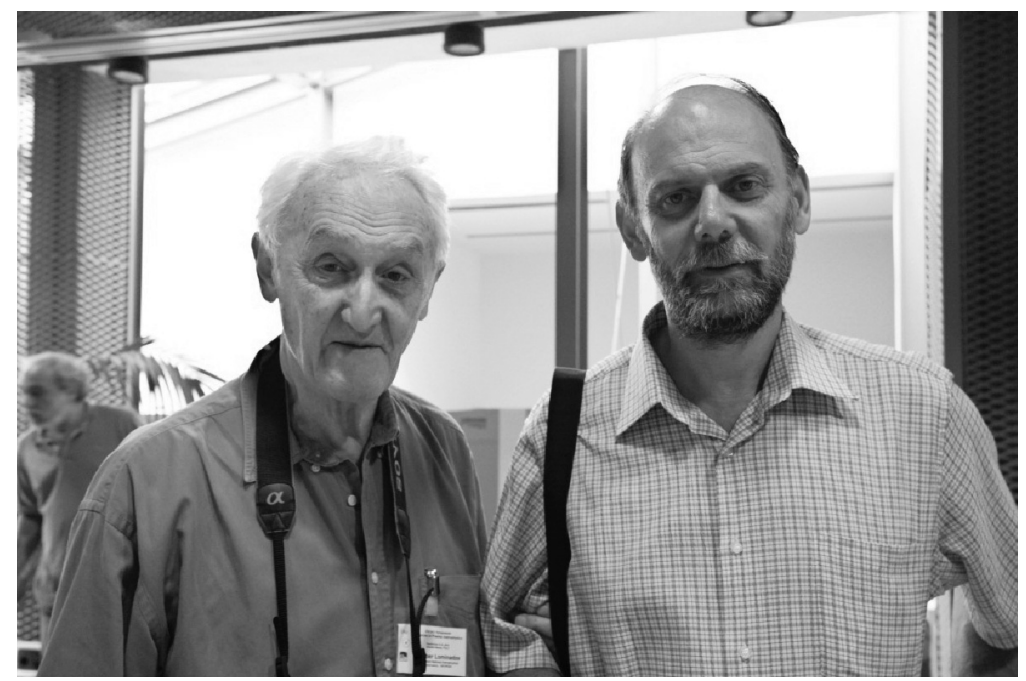

Jumber Lominadze with his son Georgi 


\section{Gerhard Haerendel}

Born in 1935, he graduated in Physics from the university of Munich in 1963. He is considered a pivotal figure in the European exploration of space, having more than 30 year of experience in space research, including the function of P.I. of several international rocket and satellite projects. His pioneering work opened a new view towards understanding of plasma in space and its interaction with the solar wind, small-scale magnetic reconnections events, high-beta plasma blobs in the magnetosphere and the in situ confirmation of reconnection, and fundamental theoretical works on basic plasma processes. He was recently awarded Jean Dominique Cassini Medal.

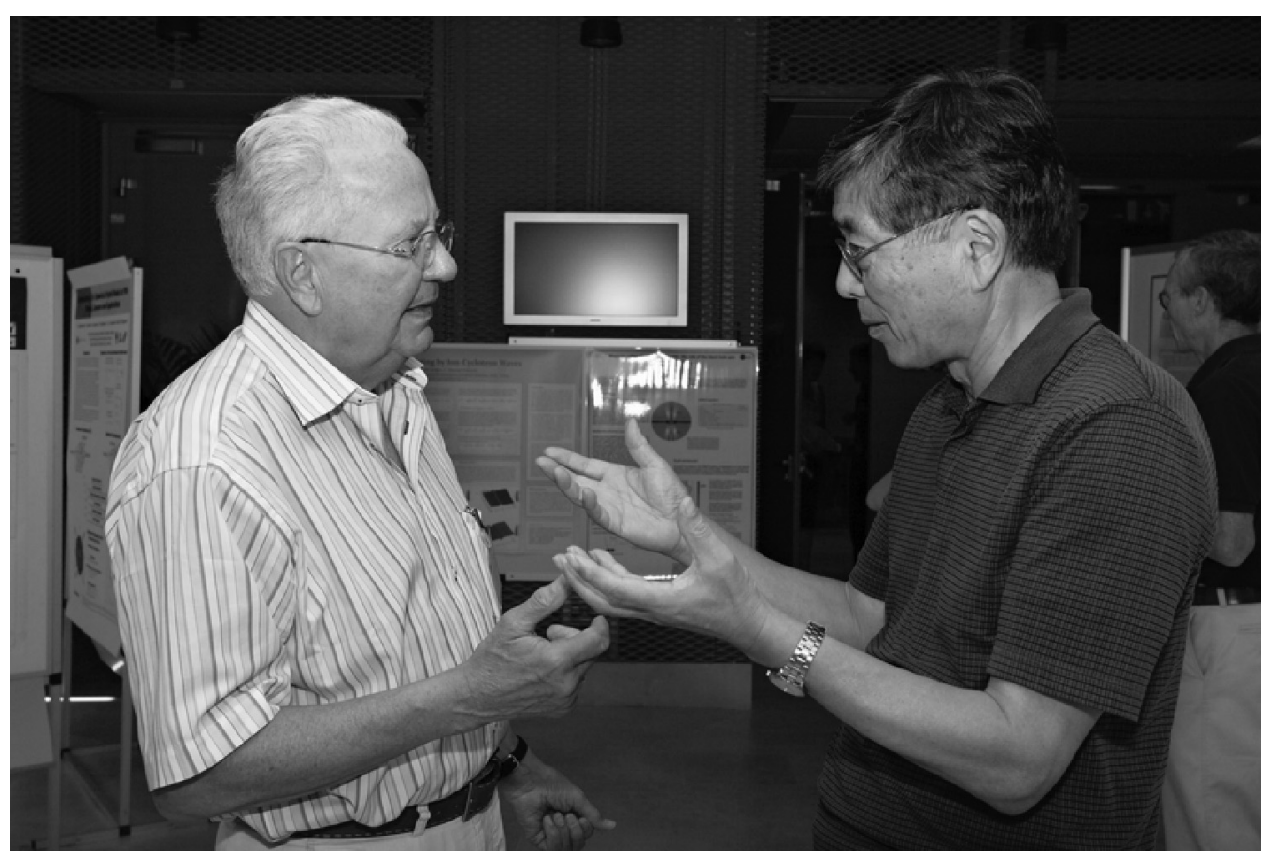

Gerhard Haerendel and Masaaki Yamada 


\section{Donald Melrose}

Professor of Theoretical Physics since 1979, Donald Melrose made specific contributions to the theory of plasma emission and its application to solar radio burst, the theory of elector cyclotron maser emission and its application to planetary and the theory of pulsar radio emission. He is recognized as one of the leading experts of kinetic theory of plasmas, plasma instabilities and nonlinear processes with application in various fields of astrophysics.

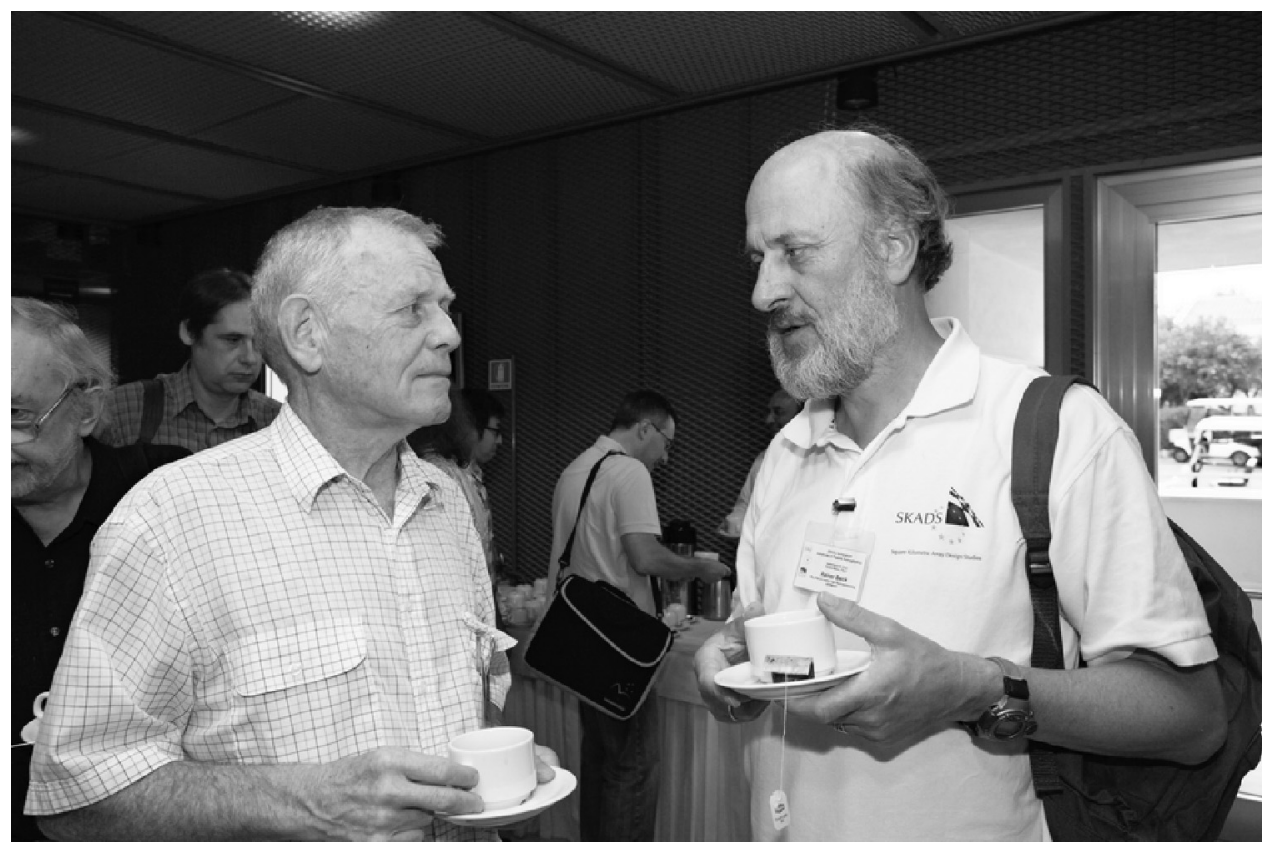

Don Melrose (left) and Rainer Beck 


\section{Lucio Paternò}

Professor at University of Catania since late 1960s, apart from a short parenthesis at Catania Astrophysical Observatory, Lucio Paternò is an outstanding figure in the Italian and international scene. His scientific activity encompasses astronomical photoelectric photometry, Solar site testing, Space physics and solar and stellar physics, in particular helioseismology and asteroseismology. He is a member of the French Academy of Sciences as well as member of the Accademia Gioenia of Natural Sciences of Catania.

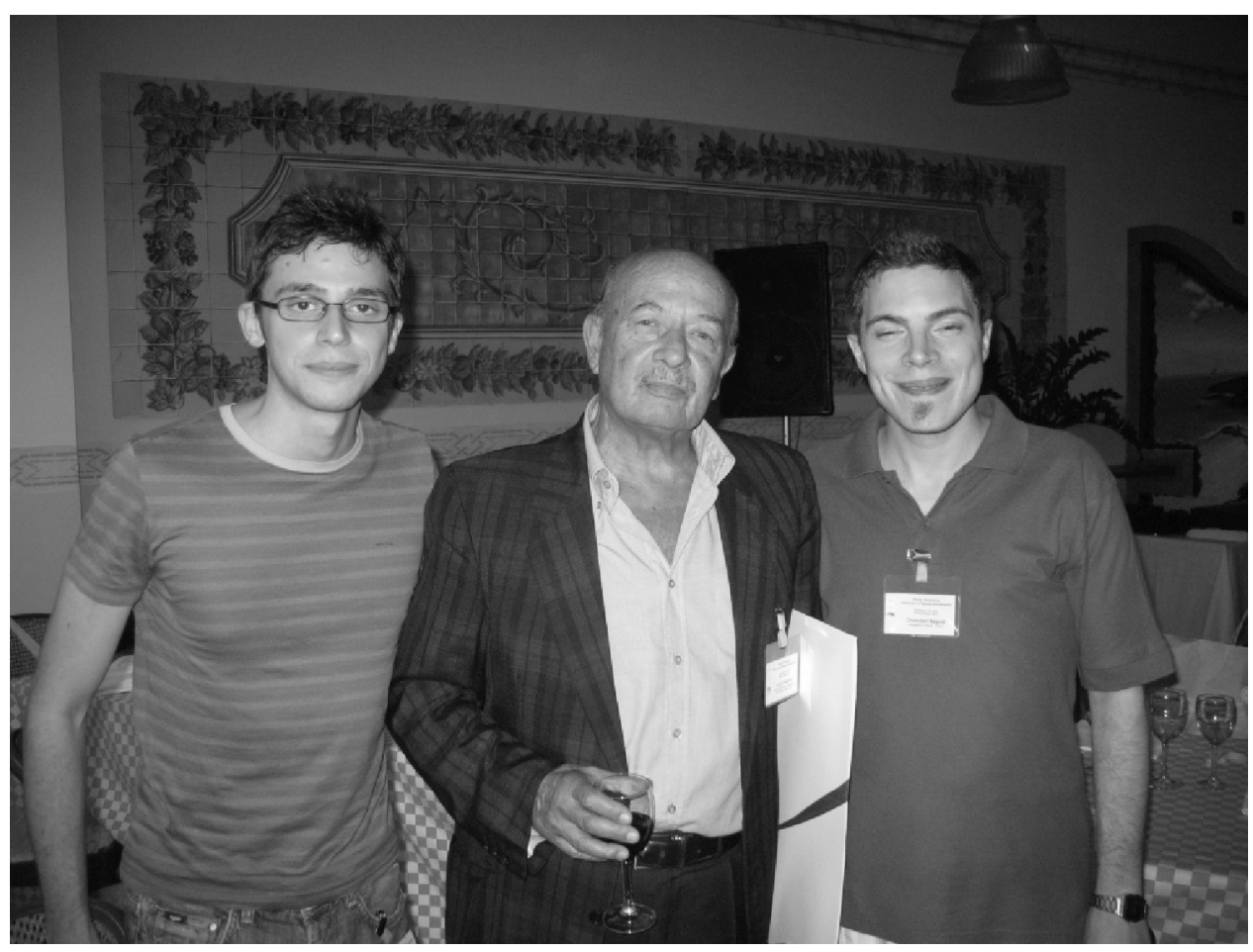

Enrico Corsaro (left), Lucio Paternò (center), and Christian Napoli (right) 


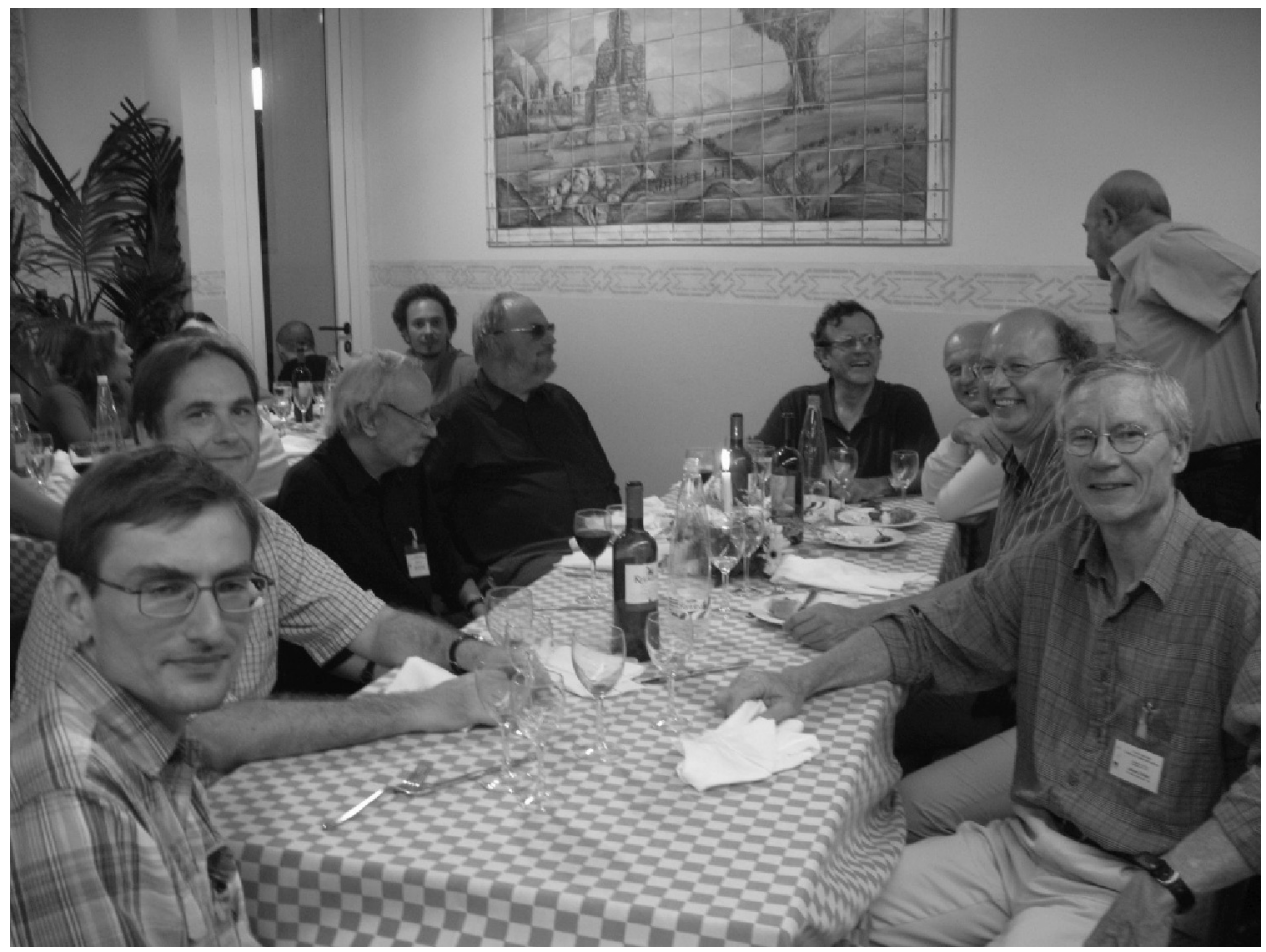

A table at the social dinner. From the right: Jim Drake, Klaus Strassmeier, Lucio Paternò, Alex Lazarian, Bob Rosner, Guenther Ruediger, Ilkka Tuominen, Jim Stone, Andrey Beresnyak 


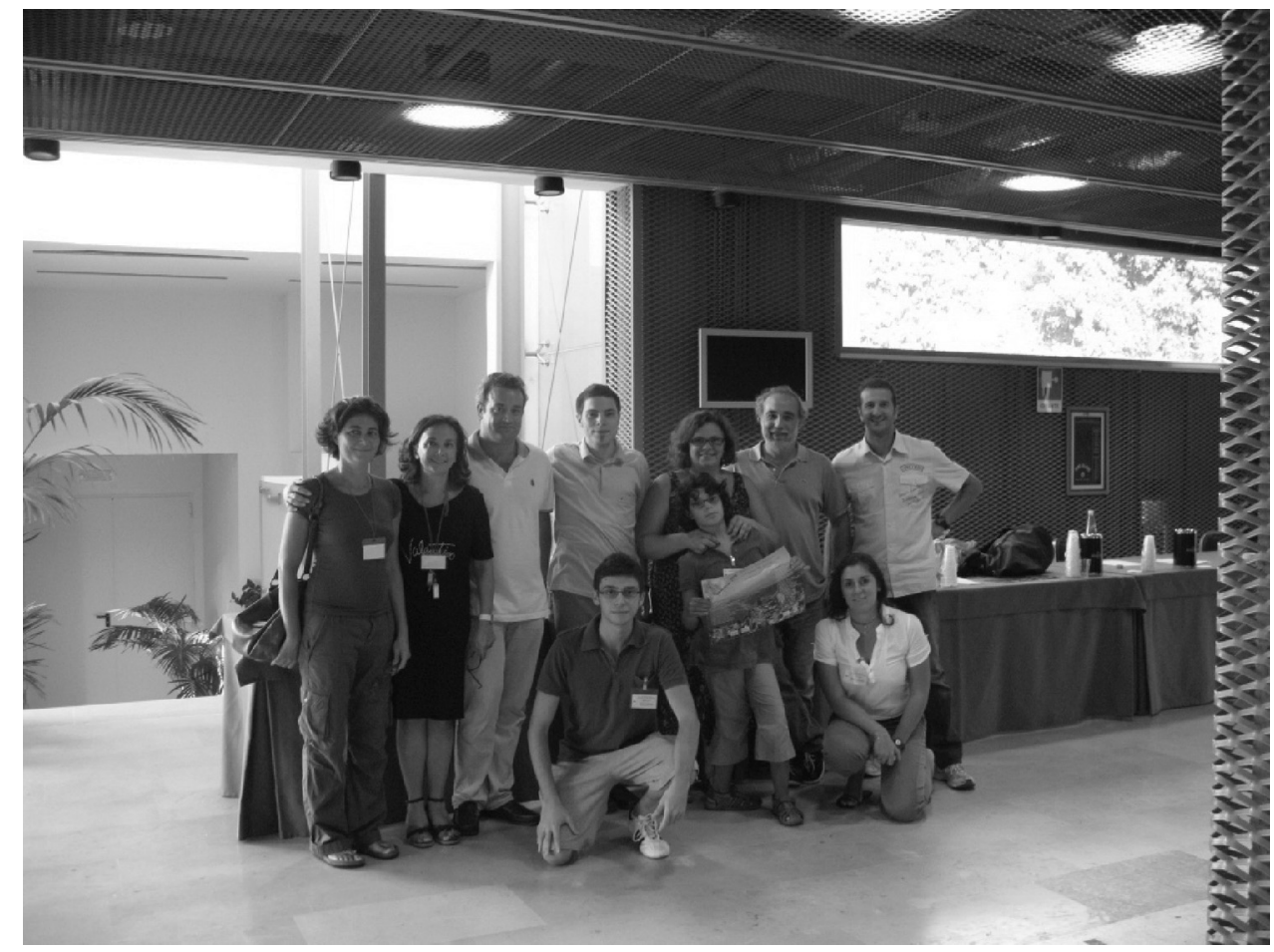

The $L O C$ 


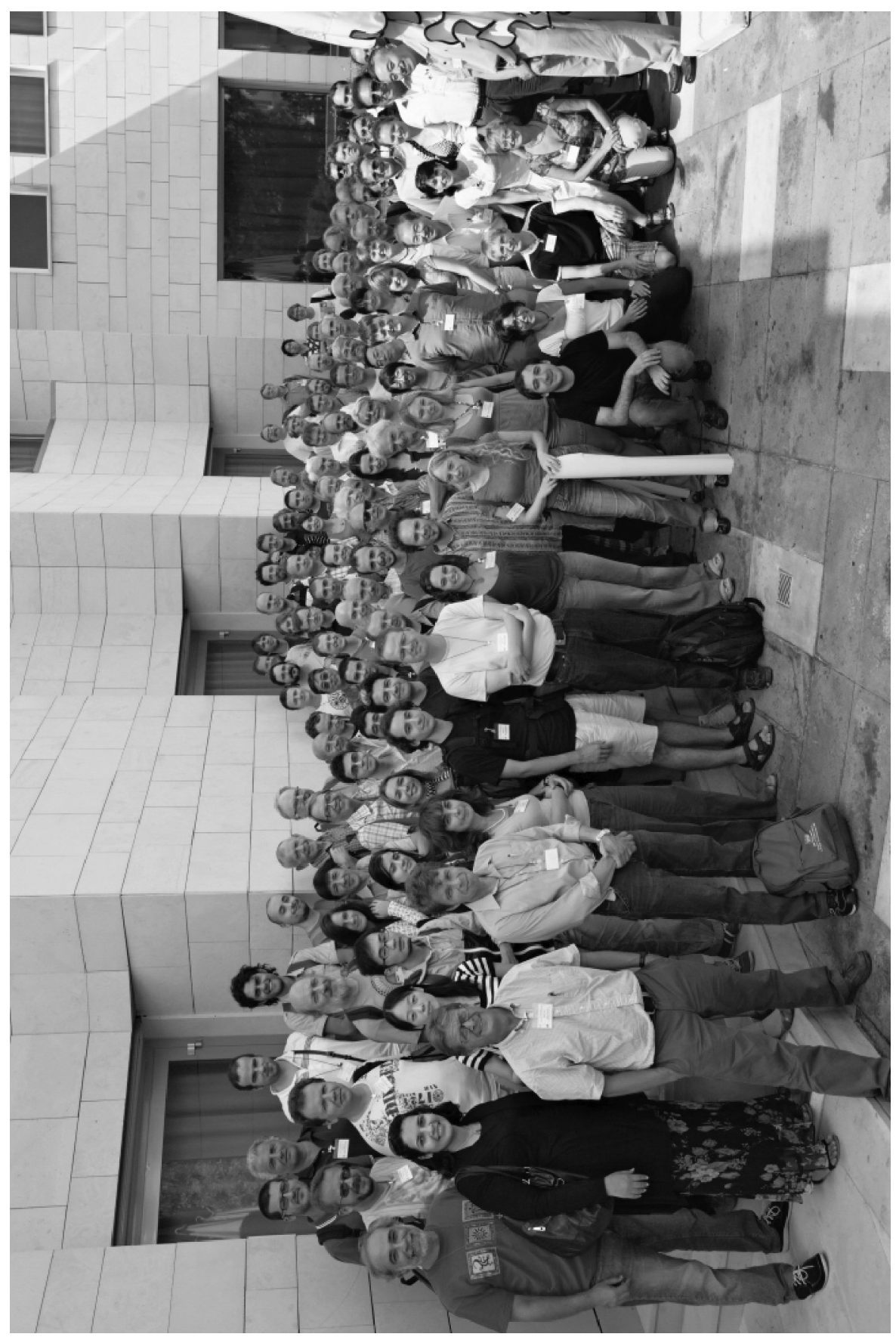

Group picture 


\section{Participants}

Antonello Anzalone INFN LSN-Catania, Italy,

Rainer Arlt Astrophysikalisches Institut Potsdam,Germany

Rainer Beck Max-Planck-Institut für Radioastronomie, Germany

Gaetano Belvedere Dept. of Physics and Astronomy, University of Catania, Italy

Svetlana Berdyugina Kiepenheuer Institut für Sonnenphysik, Germany

Andrey Beresnyak University of Wisconsin-Madison, USA

Lapo Bettarini Centre for Plasma Astrophysics, Belgium

Matteo Bocchi Imperial College London, UK

Alfio Bonanno IN AF-Osservatorio Astrofisico di Catania, Italy

Axel Brandenburg NORDITA, Sweden

Philippa Browning Jodrell Bank Centre for Astrophys., Univ. of Manchester, UK

Jörg Büchner Max-Planck-Institut für Sonnensystemforschung, Germany

Carlo Burigana INAF-IASF Bologna, Italy

Blakesley Burkhart University of Wisconsin Madison, USA

Simon Candelaresi NORDITA, Sweden

Vincenzo Capparelli Dipartimento di Fisica UNICAL, Italy

Monica Cardaci UAM \& FCAGLP, Spain

Giuseppe Castro Laboratorio Nazionale del Sud, Italy

Stirling Colgate Los Alamos National Laboratory USA

Enrico Corsaro Universitá di Catania, Italy

Neil Cramer University of Sidney, Australia

Claudio Cremaschini SISSA. Italy

Serena Dalena Universitá della Calabria, Italy

Gustavo Rocha da Silva Departamento de Astronomia - IAG/USP, Brasil

Garcia De Andrade University of Rio de Janeiro, Brasil

Elisabete de Gouveia Dal Pino Universidade de São Paulo - (IAG. -USP)

Fabio Del Sordo NORDITA, Sweden Sweden

Suzan Dogăn University of Ege, Turkey

Sandro Donato UNICAL University of Calabria, Italy

James Drake University of Maryland, USA

Anna Dubinova Institute of Applied Physics RAS, Nizhny Novgorod, Russia

Vincent Duez Argelander-Institut für Astronomie Bonn, Germany

Sergey Dyadechkin FMI, Helsinki, Finland

Natalia Dzyurkevich Max-Planck Institute for Astronomy, Germany

Rasha Emara German University in Cairo, Egypt

Adnan Erkurt Istanbul Univer., Depart. of Astr. and Space Sciences, Turkey

Luigina Feretti Inaf-IR A Bologna Italy

Attilio Ferrari Universitá di Torino, Italy

Markus Flaig Inst. for Computational Phys., Univer. of Tübingen, Germany

Gregory Fleishman New Jersey Institute of Technology, USA

Kotaro Fujisawa The University of Tokyo, Japan

Nadia Gambino I.N.F.N. Laboratori Nazionali del Sud, Italy Italy

Santo Gammino I.N.F.N. Laboratori Nazionali del Sud, Italy Italy

Urs Ganse Lehrstuhl für Astronomie, Universität Wuerzburg, Germany

Ilknur Gezer Natural and applied science, Turkey

Janusz Gil Kepler Institute of Astronomy, Zielonaga Gora, Poland

Noémie Globus Observatoire de Paris, France

Daniel OsvaldoGómez Department of Physics, University of Buenos Aires, Argentina

Antonella Greco Dipartimento di Fisica - Universitá della Calabria, Italy

Oliver Gressel Queen Mary, University of London, UK

Salvatore Guglielmino Instituto de Astrofisica de Canarias, Spain

Filippo Guarnieri University of Rome La Sapienza, Italy

Guillermo Hagele FCAGLP \& UAM, Argentina

Gerhard Haerendel Max Planck Institute for Extraterrestrial Physics, Germany

Michal Hanasz Centre for Astr., Nicolaus Copernicus University, Torun, Poland

Troels Haugbølle Niels Bohr Institute, Denmark

Mariko Hirai University of Tokyo, Japan

Subhon Ibadov Institute of Astrophysics, Tajik Academy of Sciences, Tajikistan

Stavro Ivanovski Universitá di Catania, Italy

Axel Jessner Max-Planck-Institute for Radio Astronomy, Germany

Hantao Ji Princeton University, USA

Anders Johansen Lund Observatory, Sweden

Marian Karlický Astronomical Institute, Ondrejov Observatory, Czech Republic

Subhash Chandra Kaushik School of Studies in Physics, Jiwaji Univ., India

Koen Kemel NORDITA, Sweden

Rony Keppens Centre for Plasma Astrophysics, K.U. Leuven, Belgium

Bernhard Kliem University of Potsdam, Germany

Vladimir Kocharovsky Inst. of Applied Phys., Russian Academy of Scien., Russia

Alexander Kosovichev Stanford University, USA

Manfred Küker Astrophysikalisches Institut Potsdam, Germany

Katarzyna Kulpa-Dybel Astronomical Observatory of the Jagiellonian University, Poland

Katarzyna Kulpa-Dybel Astronomical Obser

Antonino Francesco Lanza INAF-Osservatorio Astrofisico di Catania, Italy

Alex Lazarian University of Wisconsin-Madison, USA

Marcia Regina Leão Departamento de Astronomia - IAG/USP, Brasil

Sergey Lebedev Imperial College, UK

Martin Lemoine Institut d'Astrophysique de Paris, France

Fabio Lepreti Universitá della Calabria, Italy

Paolo Leto INAF - Osservatorio Astrofisico di Catania, Italy

Harald Lesch University Observatory Munich, Germany

Jumber Lominadze Abastumani National Astrophysical Observatory, Georgia

Richard Lovelace Cornell University, USA

Nazzareno Mandolesi INAF-IASF, Italy

David Mascali INFN \& Centro Sicil. di Fis. Nucl. e Strut. della Mat., Italy

Jin Matsumoto Kyoto University, Japan

William Matthaeus University of Delaware, USA

Andrew McMurry CMA, University of Oslo, Norway

Giorgi Melikidze Kepler Inst. of Astronomy, Univer. of Zielona Gora, Poland anzalone@lns.infn.it rarlt@aip.de rbeck@mpifr-bonn.mpg.de gbelvedere@ct.astro.it sveta@kis.uni-freiburg.de andrey@astro.wisc.edu

Lapo.Bettarini@wis.kuleuven.be

m.bocchi@imperial.ac.uk abonanno@oact.inaf.it brandenb@nordita.org 5 p.browning@manchester.ac.uk buechner@mps.mpg.de burigana@iasfbo.inaf.it

burkhart@astro.wisc.edu iomsn@physto.se

vincenzocapparelli@hotmail.com monica.cardaci@uam.es giuseppe.castro@lns.infn.it colgate@lanl.gov eco@oact.inaf.it

cramer@physics.usyd.edu.au cremasch@sissa.it

serena.dalena@fis.unical.it gustavords@astro.iag.usp.br garciluiz@gmail.com

dalpino@astro.iag.usp.br fadiesis@gmail.com

suzan.dogan@mail.ege.edu.tr kisspc@libero.it drake@umd.edu anndub@gmail.com

vduez@astro.uni-bonn.de egopost@gmail.com natalia@mpia.de

rasha.emara@guc.edu.eg adnan.erkurt@ogr.iu.edu.tr feretti@ira.inaf.it ferrari@ph.unito.it

flaig@tat.physik.uni-tuebingen.de

gfleishm@njit.edu
fujisawa@ea.c.u-tokyo.ac.jp 5 nadiagambino@lns.infn.it gammino@lns.infn.it

uganse@astro.uni-wuerzburg.de gezer.ilknur@gmail.com jag@astro.ia.uz.zgora.pl noemie.globus@obspm.fr gomez@iafe.uba.ar greco@fis.unical.it

o.gressel@qmul.ac.uk sgu@iac.es

guarnieri.filippo@gmail.com guille.hagele@uam.es hae@mpe.mpg.de mhanasz@astri.uni.torun.pl haugboel@nbi.dk

hirai@eps.s.u-tokyo.ac.jp

ibadovsu@yandex.ru stavro.ivanovski@gmail.com jessner@mpifr-bonn.mpg.de hji@pppl.gov anders@astro.lu.se karlicky@asu.cas.cz subash_kaushik@rediffmail.com koen@nordita.org

Rony.Keppens@wis.kuleuven.be bkliem@uni-potsdam.de kochar@appl.sci-nnov.ru sasha@sun.stanford.edu mkueker@aip.de kulpa@oa.uj.edu.pl aku@arm.ac.uk nlanza@oact.inaf.it lazarian@astro.wisc.edu mrmleao@astro.iag.usp.br s.lebedev@imperial.ac.uk lemoine@iap.fr

fabio.lepreti@fis.unical.it pleto@oact.inaf.it

lesch@usm.uni-muenchen.de contact@gsa.gov.ge

lovelace@astro.cornell.edu

mandolesi@iasfbo.inaf.it davidmascali@lns.infn.it

jin@kusastro.kyoto-u.ac.jp whm@udel.ed u andrew.mcmurry@astro.uio.no gogi@astro.ia.uz.zgora.pl 
Donald Melrose University of Sydney, Australia

Hana Mészárosová Astronomical Institute Ondrejov, Czech Republic

Natalia Minkova Tomsk State University, Russia Russia

Rosalba Miracoli INFN Laboratori Nazionali del Sud, Italy

Nishant Mittal Meerut College, India

Yosuke Mizuno UA Huntsville, USA

Guillaume Molodij Observatoire de Meudon LESIA, France

Francesco Musumeci I.N.F.N. Laboratori Nazionali del Sud, Italy

Cristian Napoli Universitá di Catania, Italy

Jacek Niemiec Institute of Nuclear Physics PAS, Poland

Giuseppina Nigro Dipartimento di Fisica UNICAL, Italy

Åke Nordlund Niels Bohr Institute, Denmark

Martin Obergaulinger Max-Planck-Institut fuer Astrophysik, Germany

Andrea Orlando Catania Astrophysical Observatory, Italy

Viktor Ostrovskiy Karpov Institute of Physical Chemistry, Russia

Katarzyna Otmianowska-Mazur Astronomical Obser. Jagiellonian Univer. Krakow, Poland

Lucio Paternó Dept. Physics \& Astronomy, University of Catania, Italy

Maria Elisabetta Palumbo INAF-Osservatorio Astrofisico di Catania, Italy

David Pascoe University of St Andrews, UK

Denise Perrone Dipartimento di Fisica UNICAL, Italy

Martin Pessah Institute for Advanced Study, USA

Gabriella Piccinelli Centro Tecnológico, FES Aragn, UNAM, Mexico

Arakel Petrosyan Space Research Inst. of the Russian Academy of Scien., Russia

John Podesta Los Alamos National Laboratory, USA

Jens Pomoell University of Helsinki, Finland

Helen Popova Moscow State University, Russia

Oliver Porth MPIA Heidelberg, Germany

Pietro Procopio Istituto di Astrofisica Spaziale sez. Bologna, Italy

Tomasz Rembiasz Max Plank Institute for Astrophysics, Garching, Germany

Maxim Reshetnyak Institute of the Physics of the Earth, Russia

Brian Reville Max-Planck-Institut fuer Kernphysik, Germany

Ronan Rochford National University of Ireland, Galway Ireland

Paolo Romano INAF-Osservatorio Astrofisico di Catania, Italy

Marina Romanova Cornell University, USA

Robert Rosner University of Chicago, USA

Ilan Roth UC Berkeley, Space Sciences, USA

Fatima Rubio da Costa University of Catania, Italy

Günther Rüdiger Astrophysikalisches Institut Potsdam , Germany

Arto Sandroos Finnish Meteorological Institute, Finland

Reinaldo Santos de Lima Departamento de Astronomia - IAG/USP, Brasil

Earl Scime West Virginia University, USA

Ildar Shaikhislamov Institute of Laser Physics SB RAS, Russia

Kazunari Shibata Kyoto University, Japan

Hubert Siejkowski Astronomical Observatory of the Jagiellonian University, Poland

Mario Scuderi Dipartimento di Fisica ed Astronomia \& INFN sez. Catania, Italy

Aimilia Smyrli University of Catania, Italy \& University of St Andrews, UK

Felix Spanier Lehrstuhl für Astronomie - Uni Wrzburg, Germany

Rodion Stepanov Institute of Continuous Media Mechanics, Russia

James M. Stone Princeton University, USA

Klaus G. Strassmeier Astrophysical Institute Potsdam,Germany

Giovanni Strazzulla INAF-Osservatorio Astrofisico di Catania, Italy

Toshiki Tajima Ludwig-Maximilians-Universität,Germany

Toshio Terasawa Institute for Cosmic Ray Research, Japan

Maurizio Ternullo INAF-Osservatorio Astrofisico di Catania, Italy

Corrado Trigilio INAF-Osservatorio Astrofisico di Catania, Italy

Enrico Maria Trotta Dipartimento di Fisica UNICAL, Italy

Yuriy Tsap Crimean Astrophysical Observatory, Ukraine

Salvatore Tudisco INFN-LNS, Italy

Ilkka Tuominen Universiy of Helsinki, Finland

Grazia Umana INAF-Osservatorio Astrofisico di Catania, Italy

Marek Vandas Astronomical Institute Ondrejov, Czech Republic

Antonio Vecchio Dipartimento di Fisica Universitá della Calabria, Italy

Loukas Vlahos Aristotle University of Thessaloniki, Greece

Miroslava Vukăcević Military Academy, Belgrade University, Serbia

Yörn Warnecke NORDITA, Sweden

Christopher Watts University of New Mexico, USA

Eli Waxman Weizmann Institute, Israel

Matthias Weidinger ITPA University of Wuerzburg, Germany

Maasaki Yamada PPPL, Princeton University, USA

Huirong Yan Kavli Institute of Astronomy and Astrophysics-PKU, China

Leonid Yasnov St.Petersburg State University, Russia

Shinichiro Yoshida University of Tokyo, Japan

Dario Zappalá INFN-Sezione di Catania

Valentina Zharkova University of Bradford, UK

Francesca Zuccarello Department of Physics and Astronomy, Italy melrose@physics.usyd.edu.au hana@asu.cas.cz nminkova@zmail.ru rosalbamiracoli@lns.infn.it nishantphysics@yahoo.com mizuno@cspar.uah.edu

guillaume.molodij@obspm.fr fmusumeci@dmfci.unict.it chnapoli@gmail.com

Jacek.Niemiec@ifj.edu.p giusy.nigro@fis.unical.it aake@nbi.dk

mobergau@mpa-garching.mpg.de aorlando@oact.inaf.i kadyshevich@mail.ru otmian@oa.uj.edu.pl lpaterno@oact.inaf.it mepalumbo@oact.inaf.it dpascoe@mcs.st-and.ac.uk denise.perrone@fis.unical.it mpessah@ias.edu

gabriela@astroscu.unam.mx apetrosy@rssi.ru

jpodesta@solar.stanford.edu

jens.pomoell@helsinki.fi

popovaelp@hotmail.com porth@mpia.d

procopio@iasfbo.inaf.it

rembiasz@mpa-garching.mpg.de

m.reshetnyak@gmail.com

brian.reville@m pi-hd.mpg.de

ronan.rochford@nuigalway.ie prom@oact.inaf.it

romanova@astro.cornell.ed

r-rosner@uchicago.ed u

ilan@ssl.berkeley.ed frdc@oact.inaf.it gruediger@aip.de

arto.sandroos@fmi.fi

rlima@astro.iag.usp.br escime@wvu.edu ildars@ngs.ru

shibata@kwasan.kyoto-u.ac.jp

h.siejkowski@oa.uj.edu.p

mario.scuderi@ct.infn.it emilia@oact.inaf.it

fspanier@astro.uni-wuerzburg.de rodion@icmm.ru

jmstone@Princeton.ED U

kstrassmeier@aip.de

gstrazzulla@oact.inaf.it

tajima.toshiki@gmail.com terasawa@icrr.u-tokyo.ac.jp

mternullo@oact.inaf.it ctrigilio@oact.inaf.it etrotta@thematica.it trotta@ yur@crao.crimea.ua tudisco@lns.infn.it

Ilkka.Tuominen@helsinki.f gumana@oact.inaf.it vandas@ig.cas.cz

antonio.vecchio@fis.unical.it

vlahos@astro.auth.gr

vuk.mira@gmail.com Joern@nordita.org cwatts@ece.unm.edu

eli.waxman@weizmann.i mweidinger@astro.uni-wuerzburg.de myamada@pppl.gov

IAU hryan@pku.edu.cn Yasnov@pobox.spbu.ru yoshida@ea.c.u-tokyo.ac.jp

dario.zappala@ct.infn.it

v.v.zharkova@brad.ac.uk fzu@oact.inaf.it 


\section{Address of the Director of Catania Astrophysical Observatory}

I am happy to give you my warmest welcome to Giardini-Naxos to attend the meeting on Plasma Astrophysics. Giardini-Naxos is today a known touristic place with a beautiful shore and a very long history. Naxos was the most ancient of all the Greek colonies in Sicily, founded in 735 BC by a body of colonists from Chalcis in Eubea. The coins of Naxos, which are of fine workmanship, may almost all be referred to the period from 460 $\mathrm{BC}$ to $403 \mathrm{BC}$, which was probably the most flourishing in the history of the city. In 403 BC, Dionysius of Syracuse determined to turn his arms against the Chalcidic cities of Sicily. He sold all the inhabitants of Naxos as slaves and destroyed both the walls and buildings of the city.

As known, the Greek culture has been particularly relevant for the development of the Sicilian culture. And, last night thinking about what to say today, I had a dream: a meeting was held in the same place where we are now but in 450 BC. I saw Empedocles (a great Sicilian philosopher/scientist, ca. 490430 BC), a progenitor of the actual organizer. Empedocles philosophy is best known for being the originator of the cosmogenic theory of the four classical elements: air (the gaseous state), water (the liquid state), earth (the solid state) and fire (the fourth state that today we call Plasma). In the dream Empedocles/Alfio organized, exactly here, a meeting on the fire/plasma. I wish( and I am pretty sure that) we all are worthy of the great history that is at the root of this land. Have a great meeting and enjoy your stay in Sicily!

Gianni Strazzulla, Director of Catania Astrophysical Observatory

Giardini-Naxos, September 2010 